\title{
Dynamic Effects May Affect Hammett Linear Free Energy Relationship
}

Xinyu Xu and Zongchang Han

[*]: Department of Chemistry, Tsinghua University, Haidian, Beijing 100084, People's Republic of China

hzc17@mails.tsinghua.edu.cn

\begin{abstract}
The aromatic nucleophilic substitution reaction in the Clayden rearrangement reaction has an abnormal Hammett linear free energy relationship ${ }^{1}$. Inspired by the concertedstepwise variation of the aromatic nucleophilic substitution reaction in $2018^{2}$, we determined the mechanism of Clayden rearrangement reaction under several conditions by DFT calculation then explained the mechanism diversity. We also found that there is a significant dynamic effect in the system due to the emergence of dynamic intermediates, which may be one of the reasons for the Hammett linear free energy relationship.
\end{abstract}

\section{Introduction}

Clayden et al. published a methodology research of amino acid synthesis in Nature at $2018{ }^{1}$ (Fig.1). The rearrangement reaction (called Clayden rearrangement) in this process has an abnormal Hammet linear free energy relationship. The reaction constant $\rho$ is significant smaller than the normal aromatic nucleophilic substitution reaction in electron-rich area and it is minus in electron-deficiency area.
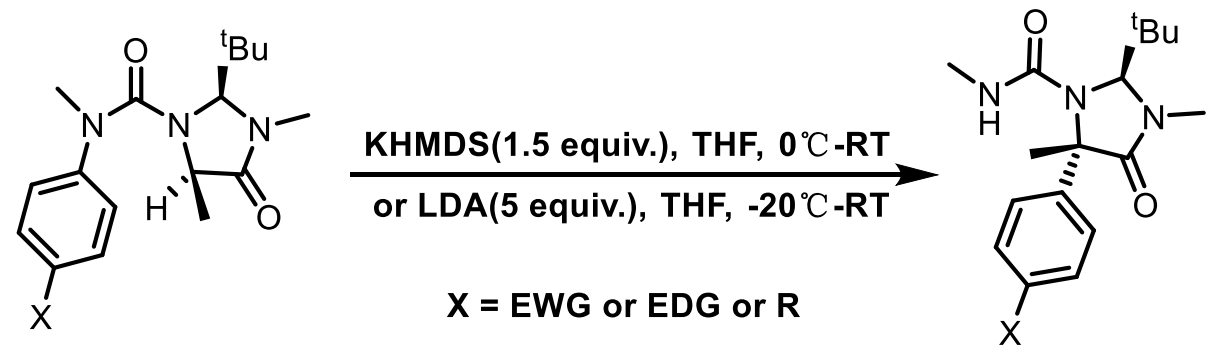

Figure 1 General Formula of Clayden Rearrangement

Clayden et al. demonstrated the existence of enol anions by experiments. The proton was extracted by the base to obtain an enol intermediate, then subjected to an aromatic nucleophilic substitution to obtain another anion intermediate. The product is obtained by work up. For the aromatic nucleophilic substitution reaction whose reaction constant is significantly smaller than normal, it is explained that the reaction mechanism will be different from the conventional aromatic nucleophilic substitution reaction mechanism and will not undergo the Meisenheimer complex(Fig. 2). However, due to lack of mechanism experiments and detailed calculation data, other intermediates have not been captured. The specific mechanism of this reaction has not yet been elucidated. 


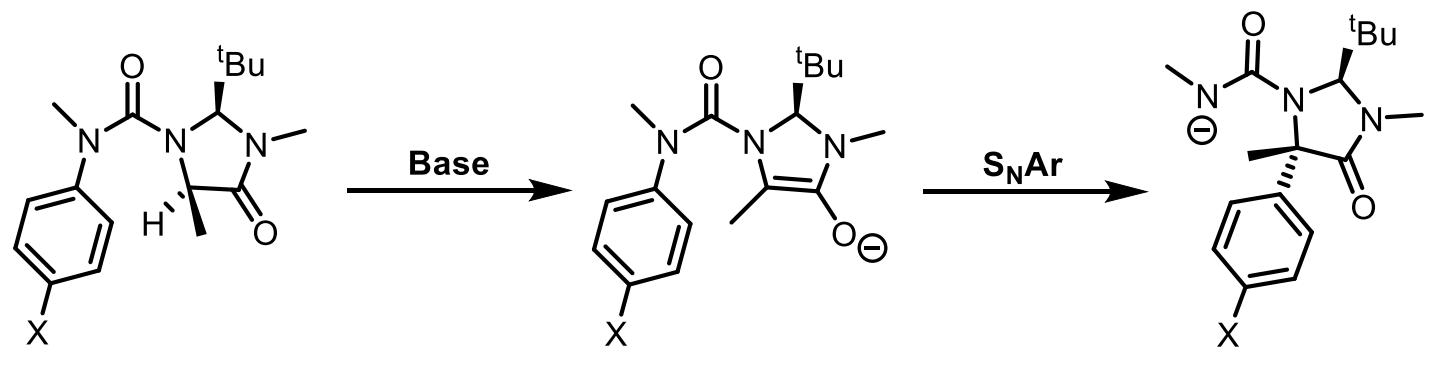

Figure 2 Confirmed Reaction Mechanism

Aromatic nucleophilic substitution reaction is a long-standing reaction between mechanism research and synthetic application. The process of addition-elimination reaction of a Meisenheimer complex has long been deeply rooted in the hearts of the people. However in 2018, the aromatic nucleophilic substitution reaction was confirmed to have three mechanisms of stepwise, concerted, and borderline types, showing different dynamic properties ${ }^{2}$. We believe that the anomalous properties of Clayden rearrangement that do not conform to the classical mechanistic model may be related to the new understanding of the concerted/stepwise property of the aromatic nucleophilic substitution mechanism.

\section{Method \& Design}

Unless otherwise stated In the calculation studies described below, all calculations use the Guassian16 B.01 software ${ }^{3}$ and the calculation level is B3LYP-D3(BJ)/def2-ma-TZVP $\mathrm{SMD}(\mathrm{THF}) / / \mathrm{B} 3 \mathrm{LYP}-\mathrm{D} 3(\mathrm{BJ}) /$ def2-SVP SMD(THF) ${ }^{4-8}$. Frequency analysis was done to check the structure is stable substance or transition state. In the experimental work, Clayden et al. used the substrates of both configurations of the carbonyl $\alpha$-position to obtain the product. S1 and S2 (Fig. 3)may have similar mechanisms due to a shared aromatic nucleophilic substitution reaction. So in order to directly compare with the experimental linear free energy relationship, the conditions used in the calculation of the linear free energy relationship will be consistent when the linear free energy relationship is determined(S2, 5equiv LDA/THF/$20^{\circ} \mathrm{C}$ ), but the reaction of $\mathbf{S} 1$ will be calculated to establish the mechanism framework to verify the available of the reaction(the extraction of proton in $\mathbf{S} 1$ is more difficult).<smiles>[X]c1ccc(N(C)C(=O)N2C(C(C)C)C(C)(C)C(=O)N2C)cc1</smiles>

S1<smiles>[X]c1ccc(N(C)C(=O)N2C(C(C)C)N(C)C(=O)[C@H]2C)cc1</smiles>

S2
$\mathrm{X}=\mathrm{OMe}, \mathrm{H}, \mathrm{CN}$

Figure 3. Structure of S1 and S2

\section{Results \& Discussion}




\section{Reaction Mechanism Framework of S1 $(X=H)$}

The calculation is based on S1 $(\mathbf{X}=\mathbf{H})$, and the free energy diagram corresponding to the mechanism of KHMDS and LDA as the base is shown in Figs. 4 and $\mathbf{5}$ below. The dotted line in the figure represents a dynamic intermediate (also found in the intermediate mechanism in the research by Jacobsen et al.). The calculations show that the reaction of S1 $(\mathrm{X}=\mathrm{H})$ with the participation of KHMDS is a classical nucleophilic addition-elimination mechanism, while the reaction with LDA participation is borderline type with the participation of dynamic intermediate.

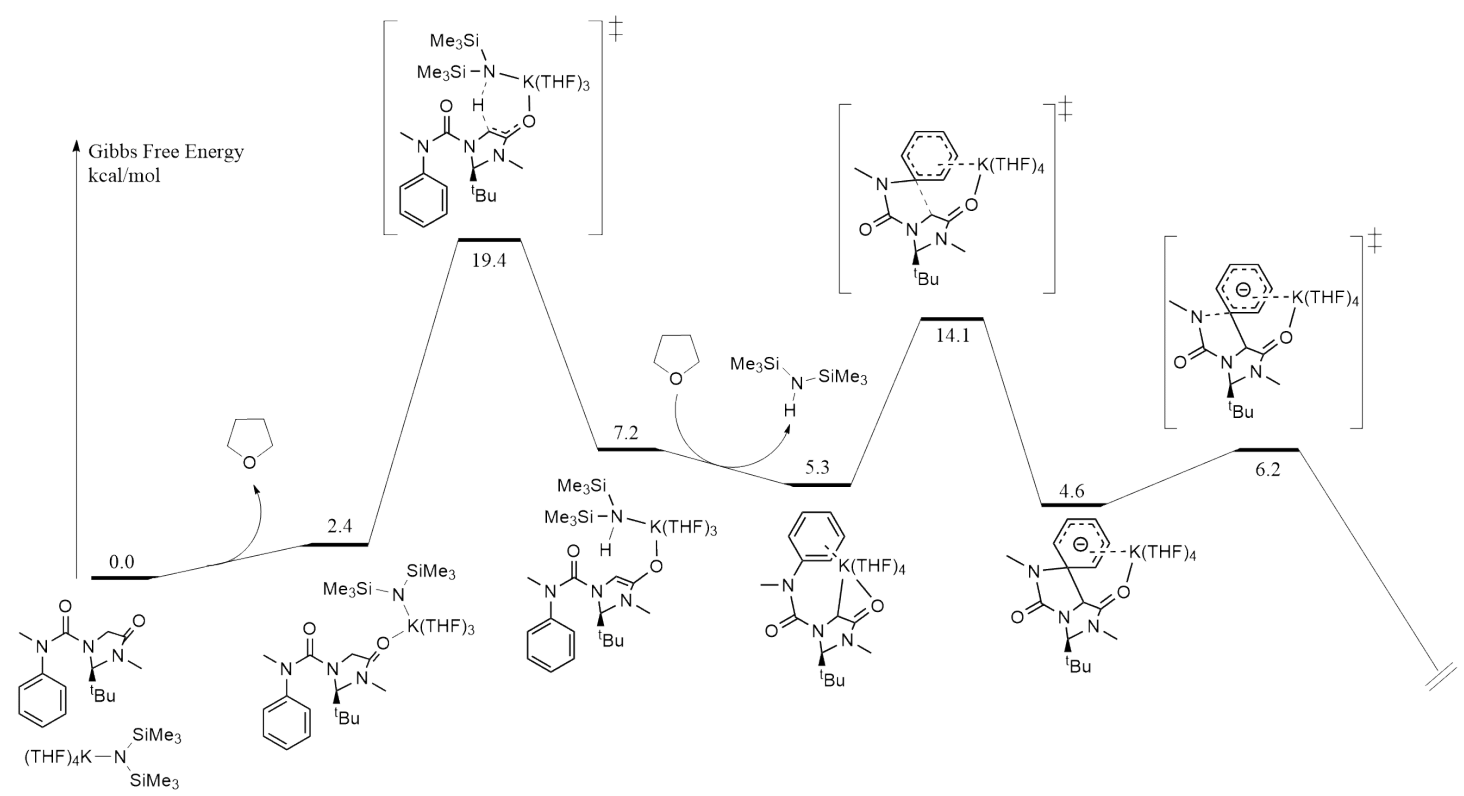

Figure 4 Reaction mechanism of $\mathbf{S} 1(X=H)$ with KHMDS

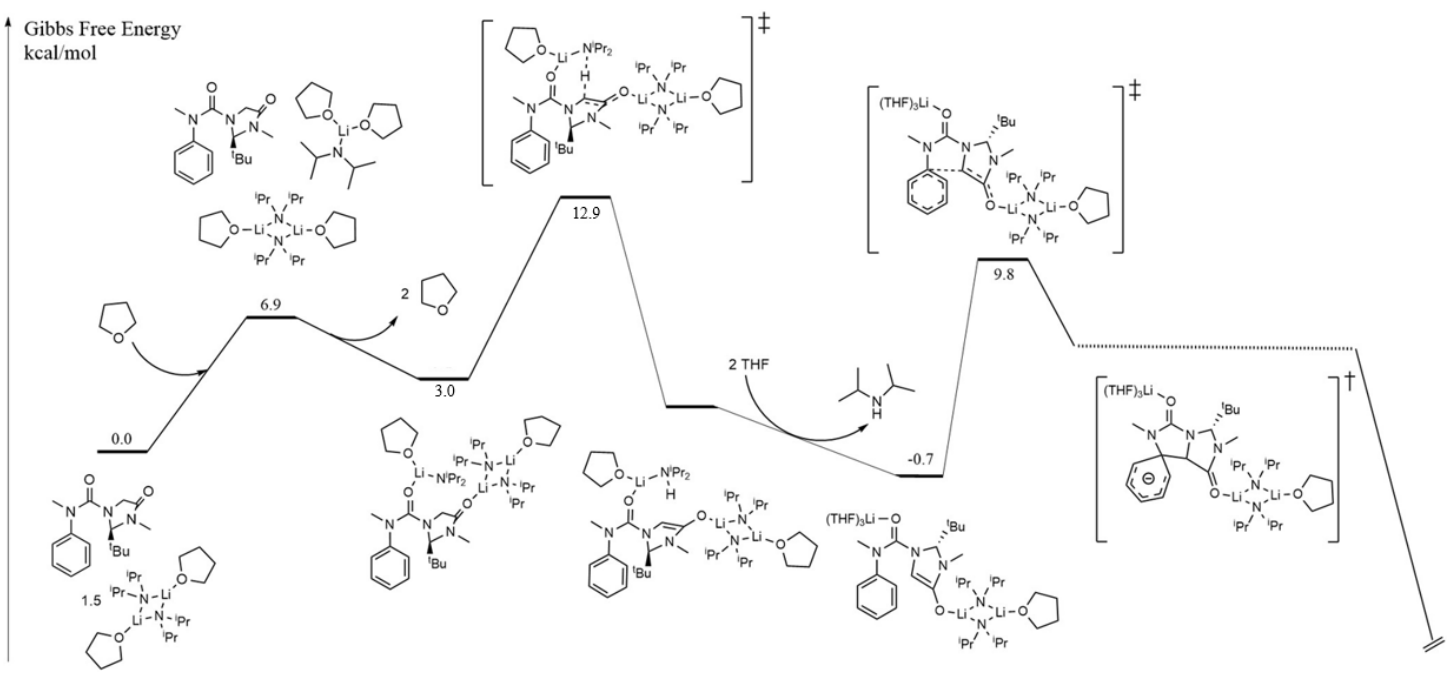

Figure 5 Reaction mechanism of $\mathrm{S} 1(\mathrm{X}=\mathrm{H})$ with LDA

\section{Discussion on Aromatic Nucleophilic Substitution Reaction}

For the enol lithium intermediate $\mathbf{A}$ obtained by deprotonation, the protonated amine in the structure can be substituted with THF to obtain another enol intermediate $\mathbf{B}$. This process is greatly exothermic. After that, the enol lithium moiety in the intermediate $\mathbf{B}$ nucleophilic 
attack on the benzene ring moiety in the molecule, this step is the final step of the aromatic nucleophilic substitution process, and its energy barrier is $10.62 \mathrm{kcal} / \mathrm{mol}$. After many attempts, we found that the corresponding Meisenheimer intermediates and dissociation transition states could not be optimized, which led us to speculate that this aromatic nucleophilic substitution reaction is likely to be a concerted mechanism with a dynamic intermediate. The results of the IRC calculations confirm the existence of dynamic intermediate which is consistent with the experimental facts reported by Clayden et al. that the Meisenheimer intermediates that are unable to intercept this reaction(Fig. 6).

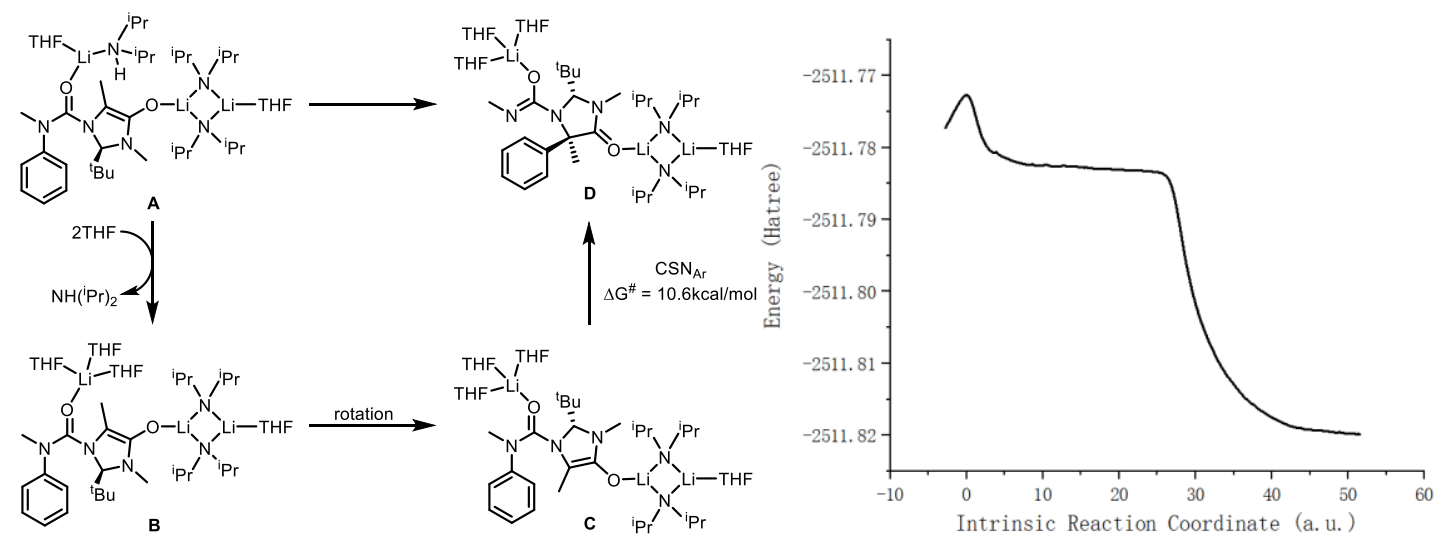

Figure 6 (1) Aromatic nucleophilic substitution reaction mechanism (Left)

(2) IRC (Right, the appearance of flat areas implies the generation of dynamic intermediate)

In the reaction involving KHMDS, the aromatic nucleophilic substitution reaction is carried out stepwise, which is a typical addition-elimination mechanism, and addition as a rate-determining step.

To approximate the linear free energy relationship and compare it with the experiment, it is necessary to separately calculate the $\mathbf{S} 2$ that $\mathbf{X}=\mathbf{C N}$ and $\mathbf{X}=\mathbf{O M e}$. Here, $\mathbf{S} 1$ was also calculated of the acid-base reaction as a reference. At this point, the potential energy surface of the entire Clayden rearrangement reaction and its apparent energy barrier can be obtained. When the substrate is $\mathbf{S 1} \mathbf{H}$ or $\mathbf{S 2} \mathbf{H}$, the rate-determining step is the acid-base reaction and the apparent energy barrier is 12.88 . Kcal/mol and $10.79 \mathrm{kcal} / \mathrm{mol}$. Based on the above calculation, the reaction mechanism and the corresponding apparent activation energy when the substituent $\mathbf{X}=\mathbf{C N}$ and $\mathbf{X}=\mathbf{O M e}$ can be obtained. When $\mathrm{X}=\mathbf{C N}$, its rate-determining step is still acid-base reaction, and the corresponding apparent energy barriers are $15.71 \mathrm{kcal} / \mathrm{mol}$ and $9.96 \mathrm{kcal} / \mathrm{mol}$ respectively (corresponding to $\mathbf{S 1} \mathbf{C N}$ and $\mathbf{S 2} \mathbf{C N}$ ). When $\mathbf{X}=\mathbf{C N}$, the aromatic nucleophilic substitution reaction is stepwise, which is also a classical additionelimination mechanism.

When $\mathrm{X}=\mathrm{OMe}$, nucleophilic substitution becomes the rate-determining step of the rearrangement reaction, and its energy barrier is $16.23 \mathrm{kcal} / \mathrm{mol}$. When $\mathbf{X}=\mathbf{O M e}$, the IRC of the aromatic nucleophilic substitution reaction also has a distinct flat zone, but there is a significant shortening compared to $\mathrm{X}=\mathrm{H}($ Fig. 7). 


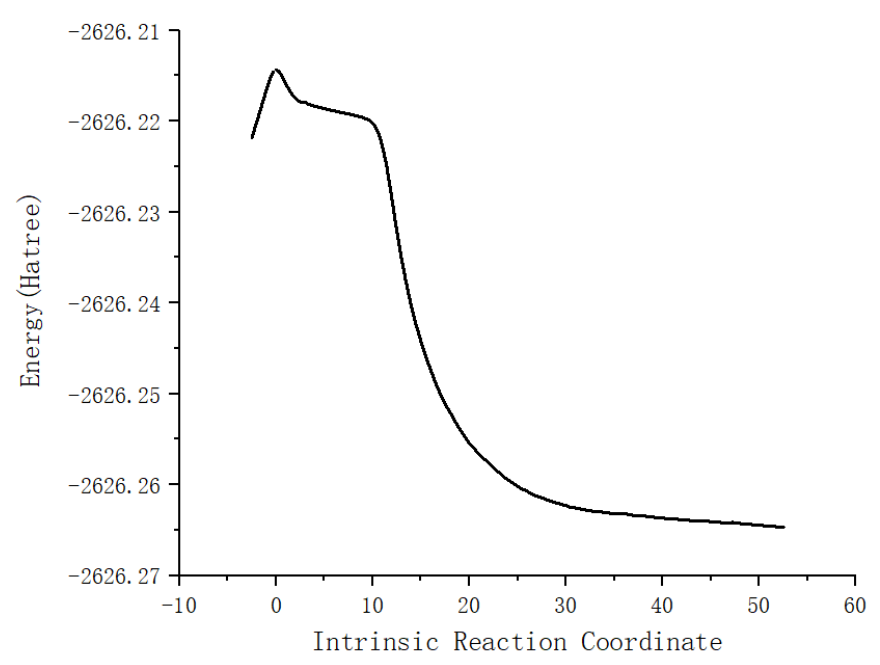

Figure 7 IRC when $\mathrm{X}=\mathrm{OMe}$

For the aromatic nucleophilic substitution step in this rearrangement reaction, when the substituent $\mathrm{X}$ is changed from $\mathrm{CN}$ to $\mathrm{OMe}$, the classical stepwise mechanism to the borderline mechanism with dynamic intermediate can be clearly observed based on IRC. This phenomenon can be explained by Marcus theory ${ }^{9}$. When the substituent changes from electron withdrawing group to electron donating group, the stability of the Meisenheimer intermediate will gradually decrease, corresponding to the gradual increase of the relative energy of the intermediate. At this time, the potential energy curve of the intermediate will intersect with the potential energy curve of the product earlier, which leads to the emergence of dynamic intermediates. Moreover, as the ability of the substituents to give electrons increases, the lifetime of the dynamic intermediate will shortened to correspond to a narrower flat area in the IRC(Fig. 8).
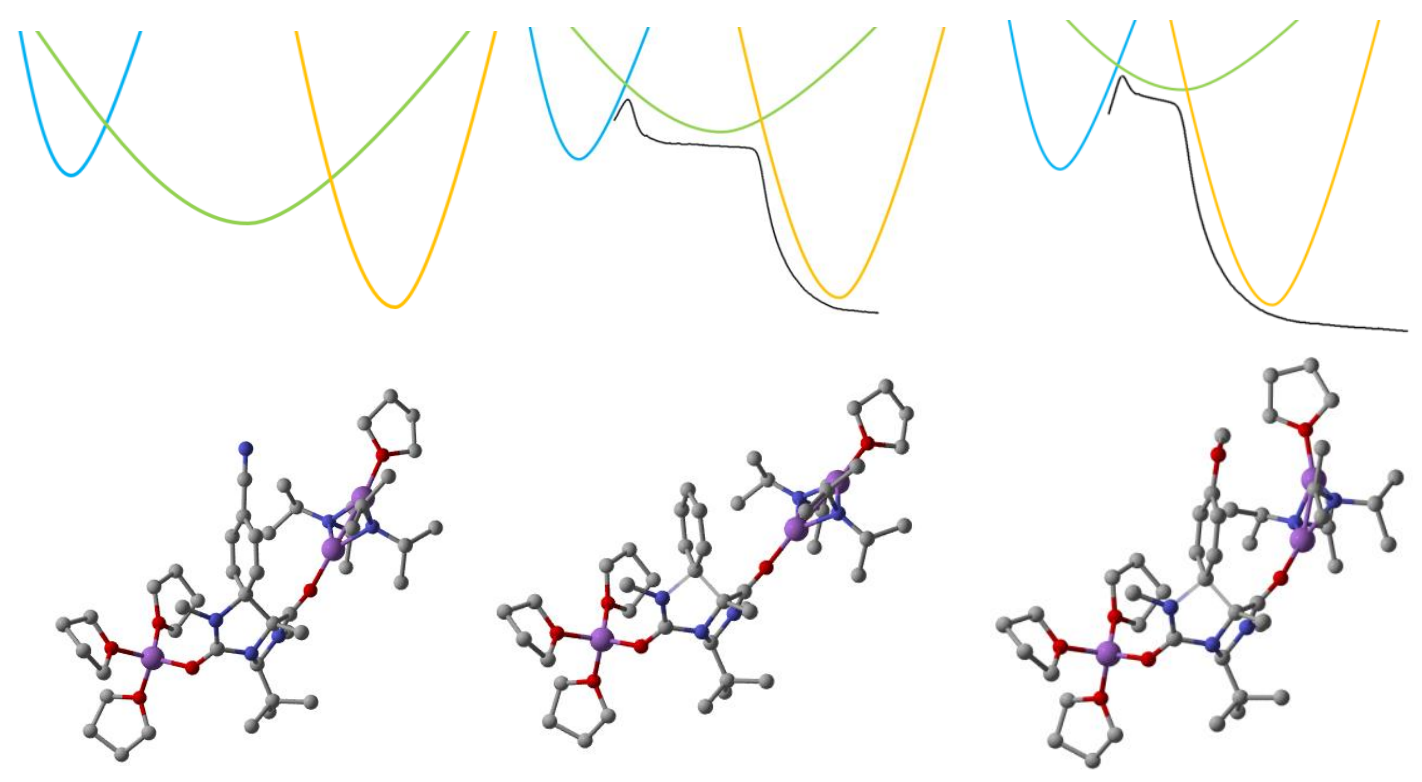

Figure 8 Explanation of Mechanism Variations with Different Substituents ${ }^{10}$

In addition, in this series of aromatic nucleophilic substitution reactions, there is another 
concerted-stepwise change.We also calculated the mechanism of aromatic nucleophilic substitution reaction under the condition of KHMDS as a base. Since only 1.5 equivalents of KHMDS were added during the reaction, the amide group in this process was not directly complexed with the metal ion. At this point, both the Meisenheimer intermediate and the dissociation transition state can be optimized, indicating that this reaction is accomplished via a classical stepwise mechanism. In this regard, we believe that here the nucleophilicity of the nucleophile and the leaving ability of the leaving group affect the mechanism. We proposes the following explanation: Approximately, the energy of the molecule depends on its most unstable part, so the energy of reactant depends on the enolate, the energy of intermediate depends on the benzene ring and the energy of product depends on the amide salt. LDA's Lewis acidity makes the enolate and amide salt more stable. Therefore, compared with KHMDS, the energy of the reactants and products is reduced while maintaining the same energy of the intermediate, which is equivalent to increasing the relative energy of the intermediate. As a result, the apparent activation energy rises and the mechanism change from stepwise to borderline, and a dynamic intermediate is present. The reason why the concerted mechanism is difficult to occur in this system is that the reaction is exothermal strongly, and the potential energy curve of the product is too low to inevitably intersect with the intermediate(Fig. 9).
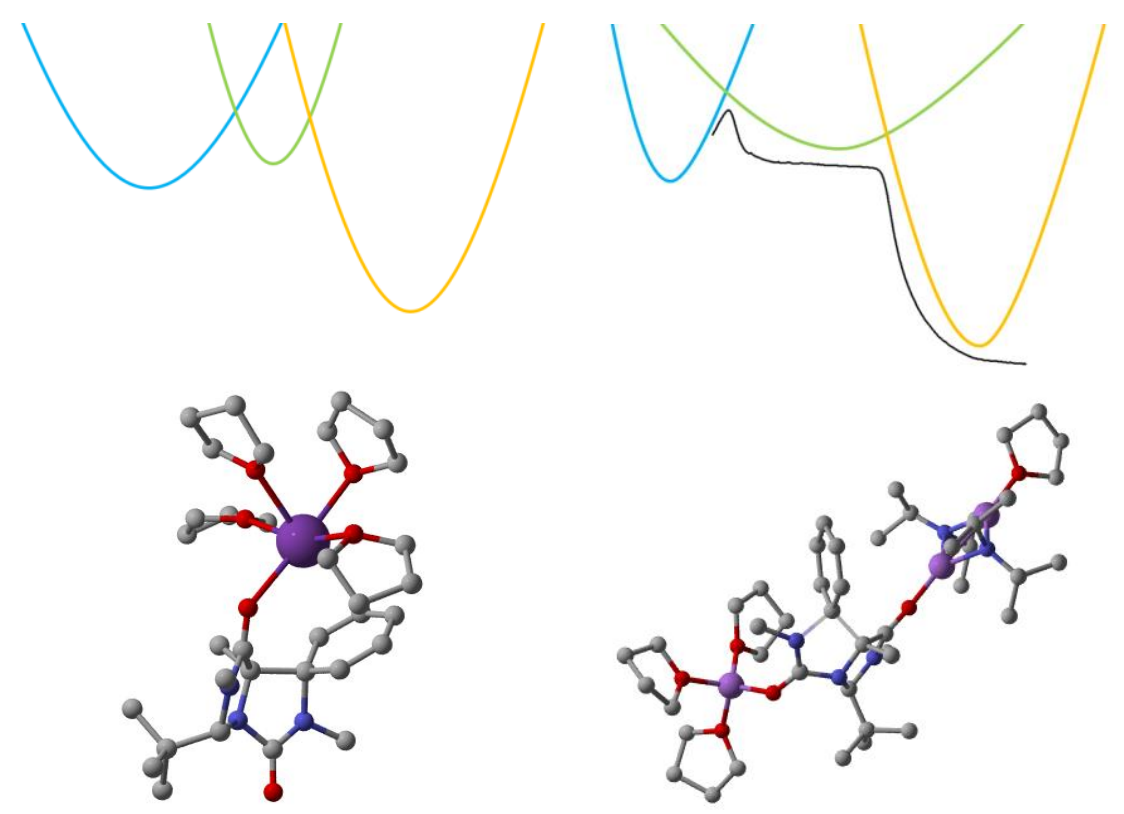

Figure 9 Effect of nucleophile and leaving group on mechanism ${ }^{10}$

The energy barrier corresponding to these three representative substituents can obtain the linear free energy relationship ${ }^{11}$ for $\mathbf{S} 1$ and $\mathbf{S} 2$ respectively(Fig. 10). For the reaction of S2, the linear free energy relationship obtained by theoretical calculation and the experimental research result (rich The electron region $\rho=4.5$, the slope of the electron-deficient region is negative), and it can be found that there is still a large difference between the two. 

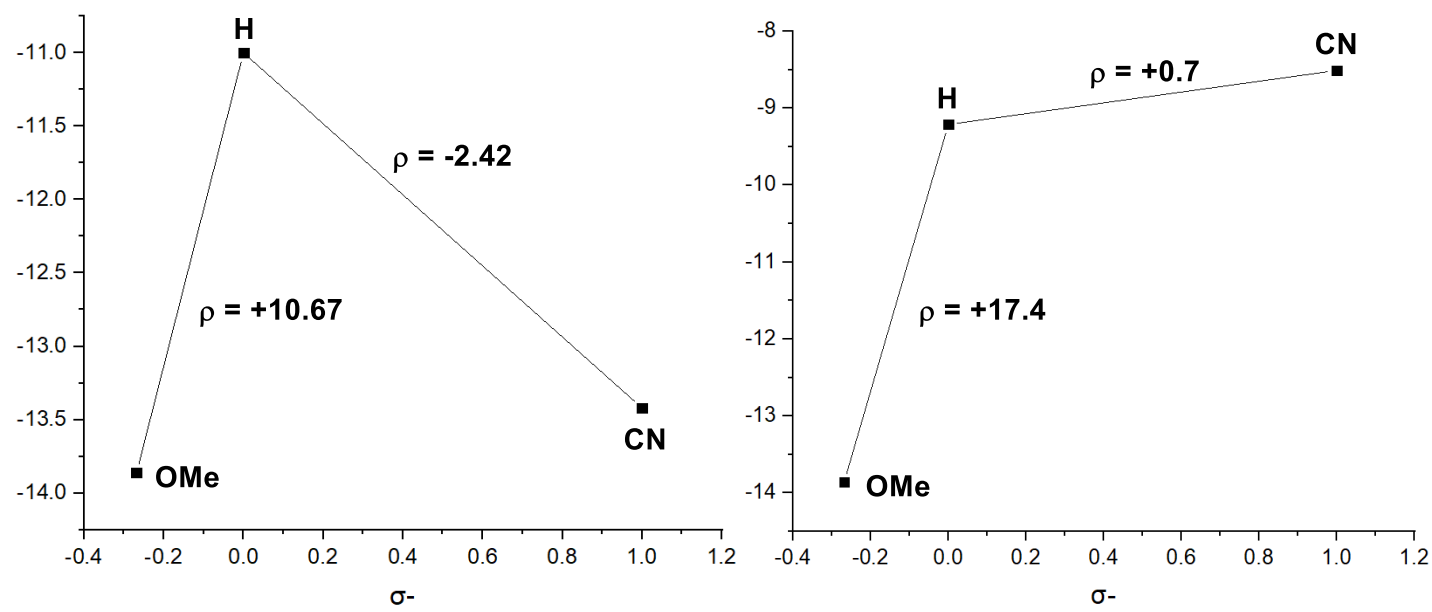

Figure 10 LFER by Computation (Left:S1,Right:S2)

In order to determine the effect of computational level on the reproducible of linear free energy relationship, we selected a concerted aromatic nucleophilic substitution reaction recently reported by Ritter et a ${ }^{12}$. Based on the reaction mechanism proposed by the author, we calculated the linear free energy relationship of this reaction at the same calculation level as before and compared it with the experimentally measured linear free energy relationship. Although the IRC also confirms the concerted mechanism of this reaction, the obtained $\rho$ value is larger compared with the experimental results which reflects the insufficient of existing theoretical methods for the calculation of the linear free energy relationship(Fig. 11). We proposed that this systematic error may be derived from the error of the existing implicit solvation model which has been reported that may play a important role ${ }^{13}$.
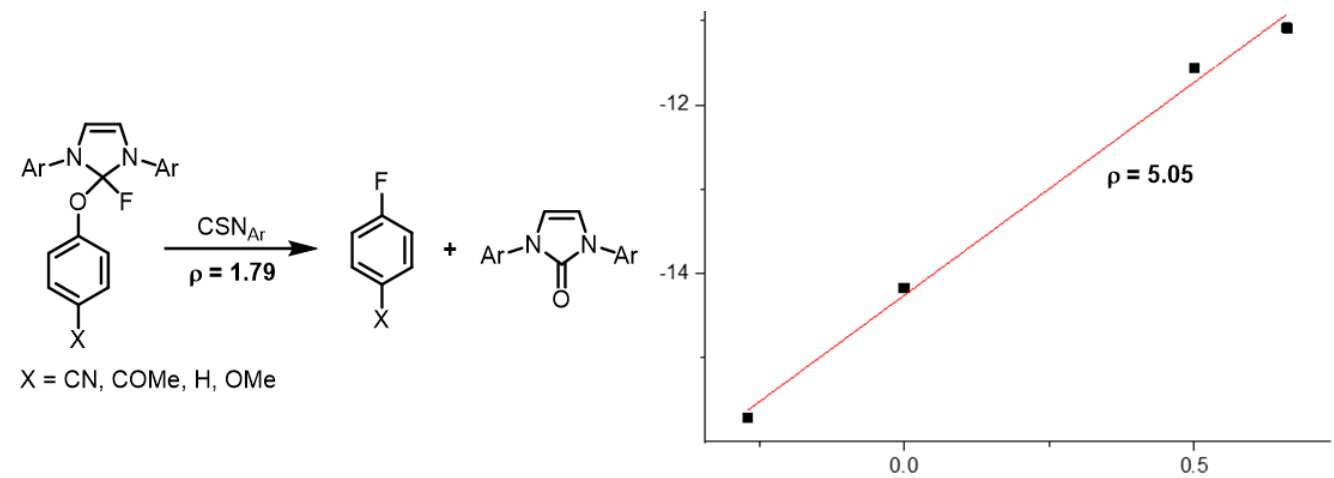

Figure 11 Calculation Error of LFER When Dynamic Effect Is Excluded

However, the difference between $\rho=1.79$ and $\rho=5.05$ seems to be very large.In fact, because the slopes of both are small, it is an acceptable deviation. When $\mathbf{X}=\mathbf{H}$ and $\mathbf{X}=\mathbf{C N}$, respectively, compared with the experimentally determined apparent activation free energy, they are actually in the range of chemical precision. Even considering the influence of such an error, it is impossible to explain the huge difference between the experimental value and the calculated value of the linear free energy relationship of the Clayden rearrangement. Considering the IRC shape described above, we suspect that this may be the effect of the dynamic effect. IRC represents the trajectory of the initial velocity of 0 , so the longer the gradual zone in the IRC, the more representative of the trajectory of the system is likely to 
stay longer in the dynamic intermediate. The rate of reaction in the presence of a dynamic intermediate will deviate from the prediction of the transition state theory due to the longer trajectory. The more stable the dynamic intermediate is, the lower the prediction will be than the transition state theory. As for the case of stable intermediates after the transition state, it is foreseen that the trajectory is longer, and the rate of the total reaction is lower than the rate of the first-step reaction predicted by the transition state theory. When the rates of the three substrates decrease to varying degrees, the Hammett relationship may change: $\mathbf{X}=\mathbf{H}$ is much lower than $\mathbf{X}=\mathbf{O M e}$, which makes the electron-rich region $\rho$ smaller; $\mathbf{X}=\mathbf{C N}$ is much lower than $\mathbf{X}=\mathbf{H}$. , the slope of the electron-deficient zone may become negative(Fig. 12).

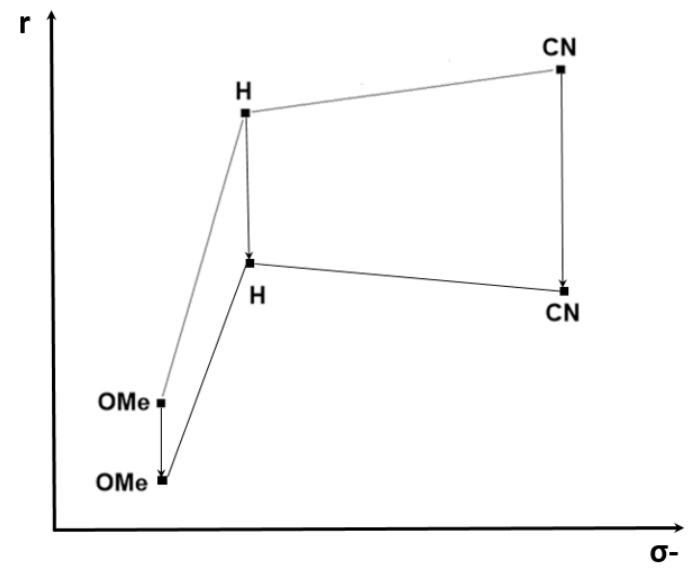

Figure 12 Schematic Diagram of the Dynamic Effect Affecting LFER

In order to confirm the existence of this dynamic effect, we used the quasi-classical molecular dynamic software PROGDYN ${ }^{14}$ to simulate the reaction when $\mathrm{X}=\mathrm{H}$ and $\mathrm{X}=\mathrm{OMe}$, and investigated the dynamics effect through the shape of the trajectory. Due to limited computing power, we simplified the group complexed with cyclic amide $\mathrm{O}$ to dimethylamino lithium and the $\mathrm{O}$ complexing group of urea to lithium ion. The trajectory shapes of the two compounds are similar. We scheme a typical set to compare(Fig. 13).
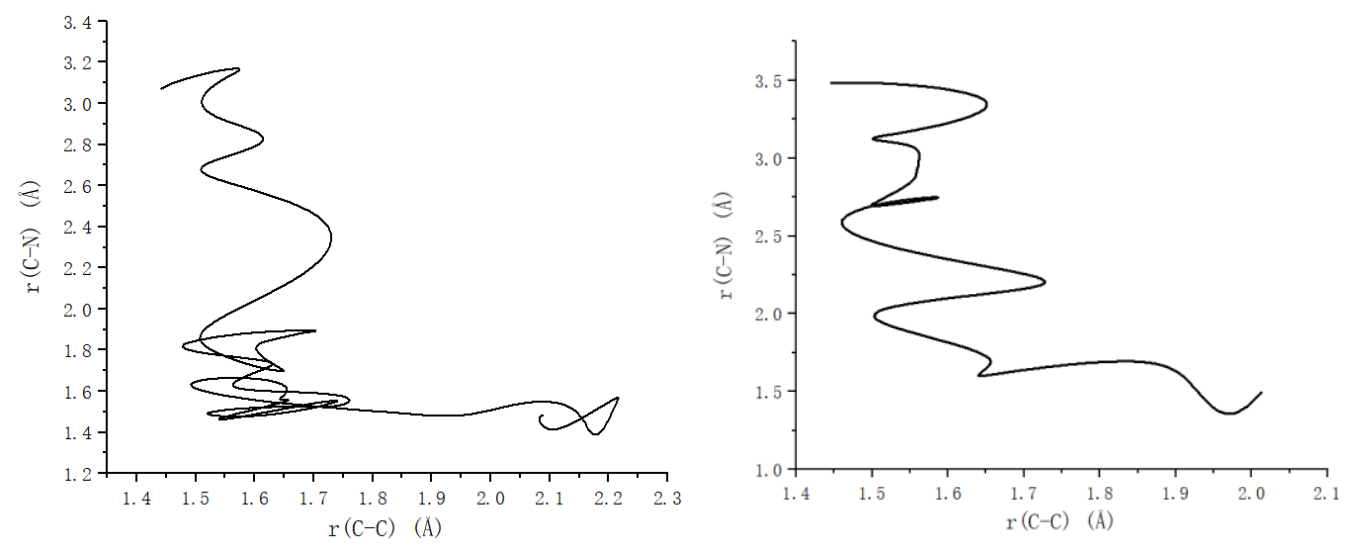

Figure 13 A Trajectory when $\mathrm{X}=\mathrm{H}$ (Left) and $\mathrm{X}=\mathrm{OMe}$ (Right)

It can be seen that when $\mathbf{X}=\mathbf{H}$, the system will stay at the dynamic intermediate for longer than $\mathbf{X}=\mathbf{O M e}$, and the other trajectories basically conform to this phenomenon. We believe that in this system with a gradual change in mechanism, the dynamics effect may be one of 
the reasons why the reaction constant is smaller than the classical stepwise or concerted reaction. However, since the real system is far more complicated than the model used for calculation, we still cannot assert that this is decisive, and only one possibility is proposed here. We will continue the research and look forward to further arguments and counterarguments.

\section{Acknowledgement}

Thanks for supporting of Tsinghua Xuetang Talents Program and guiding from Yilei Wang. And we are also appreciated to get inspiring discussions at THUchemSAST (Students' Association of Science and Technology, Department of Chemistry, Tsinghua Univ.).Finally, both authors are fans of FLOWERS, so we are appreciated to its creator Miki Sugina et al. and their company Innocent Grey. We wish that their new production Kara No Shoujo the Third Episode master up successfully soon.

\section{Reference}

(1) Leonard, D. J.; Ward, J. W.; Clayden, J. Asymmetric $\alpha$-arylation of amino acids. Nature 2018, 562, 105-109.

(2) Kwan, E. E.; Zeng Y.; Besser, H. A.; Jacobsen, E. N. Concerted nucleophilic aromatic substitutions. Nat. Chem. 2018, 10, 917-923.

(3) Gaussian 16, Revision B.01, M. J. Frisch, G. W. Trucks, H. B. Schlegel, G. E. Scuseria, M. A. Robb, J. R. Cheeseman, G. Scalmani, V. Barone, G. A. Petersson, H. Nakatsuji, X. Li, M. Caricato, A. V. Marenich, J. Bloino, B. G. Janesko, R. Gomperts, B. Mennucci, H. P. Hratchian, J. V. Ortiz, A. F. Izmaylov, J. L. Sonnenberg, D. Williams-Young, F. Ding, F. Lipparini, F. Egidi, J. Goings, B. Peng, A. Petrone, T. Henderson, D. Ranasinghe, V. G. Zakrzewski, J. Gao, N. Rega, G. Zheng, W. Liang, M. Hada, M. Ehara, K. Toyota, R. Fukuda, J. Hasegawa, M. Ishida, T. Nakajima, Y. Honda, O. Kitao, H. Nakai, T. Vreven, K. Throssell, J. A. Montgomery, Jr., J. E. Peralta, F. Ogliaro, M. J. Bearpark, J. J. Heyd, E. N. Brothers, K. N. Kudin, V. N. Staroverov, T. A. Keith, R. Kobayashi, J. Normand, K. Raghavachari, A. P. Rendell, J. C. Burant, S. S. Iyengar, J. Tomasi, M. Cossi, J. M. Millam, M. Klene, C. Adamo, R. Cammi, J. W. Ochterski, R. L. Martin, K. Morokuma, O. Farkas, J. B. Foresman, and D. J. Fox, Gaussian, Inc., Wallingford CT, 2016.

(4) (a) Becke, A. D. Density-functional thermochemistry. III. The role of exact exchange. J. Chem. Phys. 1993, 98, 5648-5652 (b) Lee, C.; Yang, W.; Parr, R. G. Development of the Colle-Salvetti correlation-energy formula into a functional of the electron density. Phys. Rev. B: Condens. Matter Mater. Phys. 1988, 37, 785-789.

(5) (a) Grimme, S.; Antony, J.; Ehrlich, S.; Krieg, H. A consistent and accurate ab initio parametrization of density functional dispersion correction (DFT-D) for the 94 elements H-Pu. J. Chem. Phys. 2010, 132, 154104-154119. (b) Grimme, S.; Ehrlich, S.; Goerigk, L. Effect of the damping function in dispersion corrected density functional theory. J. Comput. Chem 2011, 32, 1456-1465.

(6) Weigend, F.; Anlrichs, R. Balanced basis sets of split valence, triple zeta valence and quadruple zeta valence quality for $\mathrm{H}$ to Rn: Design and assessment of accuracy. Phys. Chem. Chem. Phys. 2005,7, 3297-3305

(7) Zheng, J.; Xu, X.; Truhlar, D. G.; Minimally augmented Karlsruhe basis sets. J. Chem. 
Theory Comput. 2011, 128, 295-305.

(8) Marenich, A. V.; Cramer, C. J.; Truhlar, D. G. Universal solvation model based on solute electron density and on a continuum model of the solvent defined by the bulk dielectric constant and atomic surface tensions. J. Phys. Chem. B 2009, 113, 63786396.

(9) (a) Marcus, R. A.; Sutin, N. Electron transfers in chemistry and biology. Biochim. Biophys. Acta 1985, 811, 265-322 . (b) Kwan, E. E.; Zeng Y.; Besser, H. A.; Jacobsen, E. N. Concerted nucleophilic aromatic substitutions. Nat. Chem. 2018, 10, 917-923.

(10) C. Y. Legault, CYLview, Université de Sherbrooke, 2009

(11) (a) Hammett, L. P. The Effect of Structure upon the Reactions of Organic Compounds. Benzene Derivatives. J. Am. Chem. Soc. 1937, 59, 96-103. (b) Hansch, C.; Leo, A.; Taft, R. W. A survey of Hammett substituent constants and resonance and field parameters. Chem. Rev. 1991, 91, 165-195.

(12) Neumann, C. N.; Hooker, J. M.; Ritter, T. Concerted nucleophilic aromatic substitution with ${ }^{19} \mathrm{~F}^{-}$and ${ }^{18} \mathrm{~F}^{-}$. Nature 2016, 534, 369-373.

(13) Ma, Yumiao. Solvent Molecules Play a Role in an SNAr Reaction. ChemRxiv. 2019 Preprint.

(14) Daniel A. Singleton,*, Chao Hang,Michael J. Szymanski, and, and Erin E. Greenwald Journal of the American Chemical Society 2003 125 (5), 1176-1177 